\title{
Heavy-Metal-Induced Nephropathy
}

National Cancer Institute

\section{Source}

National Cancer Institute. Heavy-Metal-Induced Nephropathy. NCI Thesaurus. Code C123028.

Damage to the kidney and renal tubules resulting from heavy metal exposure. 